\title{
The Estimated Incidence of Induced Abortion In Ethiopia, 2008
}

\section{By Susheela Singh, Tamara Fetters, Hailemichael Gebreselassie, Ahmed Abdella, Yirgu Gebrehiwot, Solomon Kumbi and Suzette \\ Audam}

Susheela Singh is vice president for research and Suzette Audam is research associate/ data manager, both with the Guttmacher Institute, New York. Tamara Fetters is senior associate, Ipas, Chapel Hill, NC,

USA. Hailemichael Gebreselassie is senior research advisor, Ipas Africa Alliance, Nairo-

bi, Kenya. Solomon Kumbi is associate professor of obstetrics and gynecology, and Ahmed Abdella and Yirgu Gebrehiwot are consultant obstetrician/gynecologists-all with Addis Ababa University, Addis Ababa, Ethiopia.

CONTEXT:Unsafe abortion is an important health problem in Ethiopia; however, no national quantitative study of abortion incidence exists. In 2005, the penal code was revised to broaden the indications under which induced abortion is legal. It is important to measure the incidence of legal and illegal induced abortion after the change in the law.

METHODS: A nationally representative survey of a sample of 347 health facilities that provide postabortion or safe abortion services and a survey of 80 professionals knowledgeable about abortion service provision were conducted in Ethiopia in 2007-2008. Indirect estimation techniques were applied to calculate the incidence of induced abortion. Abortion rates, abortion ratios and unintended pregnancy rates were calculated for the nation and for major regions.

RESULTS: In 2008, an estimated 382,000 induced abortions were performed in Ethiopia, and 52,600 women were treated for complications of such abortions. There were an estimated 103,000 legal procedures in health facilities nationwide- $27 \%$ of all abortions. Nationally, the annual abortion rate was 23 per 1,000 women aged 15-44, and the abortion ratio was 13 per 100 live births. The abortion rate in Addis Ababa (49 per 1,000 women) was twice the national level. Overall, about $42 \%$ of pregnancies were unintended, and the unintended pregnancy rate was 101 per 1,000 women.

CONCLUSIONS: Unsafe abortion is still common and exacts a heavy toll on women in Ethiopia. To reduce rates of unplanned pregnancy and unsafe abortion, increased access to high-quality contraceptive care and safe abortion services is needed.

International Perspectives on Sexual and Reproductive Health, 2010, 36(1):16-25.

As part of law reform in Ethiopia in 2005, the penal code was revised to broaden the indications under which abortion is permitted. Termination of pregnancy is now legal when the pregnancy results from rape or incest, when continuation of the pregnancy endangers the health or life of the woman or the fetus, in cases of fetal impairment, for women with physical or mental disabilities, for minors who are physically or psychologically unprepared to raise a child and in cases of grave and imminent danger that can be averted only through immediate pregnancy termination. These are significant changes from the previous law, which permitted abortion only in cases of grave and imminent danger that could be averted only through immediate pregnancy termination

A second, related change was the 2006 publication by the Ministry of Health of technical and procedural guidelines for the provision of safe abortion services, to "translate the law into actionable measures" and to inform "women, health professionals, law enforcement agencies and all sectors of society" about implementation of the law. ${ }^{1}$ However, various factors-the need to inform women and providers about the new law, a shortage of trained personnel and inadequacies in the country's health care infrastructure, especially in terms of coverage of the large rural population-mean that providing wide- spread access to new services would take time and require efforts from many institutions and sectors of the population.

Prior studies have documented that unsafe abortion has been an important and ongoing health problem in Ethiopia. The 2005 Ethiopian Demographic and Health Survey (DHS) estimates that 673 women died of pregnancy-related causes for every 100,000 live births in the six years prior to the survey. ${ }^{2}$ The World Health Organization (WHO) estimates that in Eastern Africa, unsafe abortion accounts for one in seven maternal deaths. ${ }^{3}$ In a 2001-2002 study in a major university hospital in Addis Ababa, postabortion complications were one of the three leading causes of maternal mortality. ${ }^{4}$ According to a largescale study in 2000 of 15 hospitals in nine of the country's 11 regions, more than half of women treated for complications of induced abortion had gone to an untrained provider or had induced the abortion themselves. ${ }^{5} \mathrm{~A}$ bibliographic review spanning 1985-2000 indicates that many attitudinal and organizational barriers prevented women from obtaining postabortion services without delay and that these barriers resulted in low-quality postabortion services. ${ }^{6}$

According to the 2000 and 2005 DHS surveys, the level of unintended pregnancy in Ethiopia is high and may be 
increasing. ${ }^{7,8}$ The desire for smaller families is increasing, which reflects broader social and economic changes in the country: The average desired family size declined from 4.9 in 2000 to 4.0 in 2005. And although contraceptive use has increased, unmet need for contraception has remained high. Women in Addis Ababa and other urban areas are delaying marriage into their 20 s, probably in response to adverse economic conditions. ${ }^{9,10}$ This delay in marriage may result in increased sexual activity among unmarried young women, raising their risk of unintended pregnancy as well as abortion, given that childbearing outside of marriage is highly stigmatized. In DHS surveys, few unmarried women report ever having been sexually active, but data from other, smaller-scale studies suggest that sexual activity among the unmarried is not uncommon. ${ }^{11-13}$

The present study was undertaken to estimate abortion incidence in Ethiopia, to shed light on the extent to which unsafe abortion is occurring and to estimate the level of use of legal abortion procedures following the 2005 change in the abortion law and the 2006 Ministry of Health safe abortion guidelines. Better documentation of the overall incidence of abortion and of its two components-clandestine or unsafe abortion and legal abortion-is essential for informing policy decisions and program design, and for monitoring the impact of existing policies and programs in Ethiopia. Data on the overall incidence of induced abortion is a crucial indicator of women's and couples' difficulties in preventing unintended pregnancies, and of their need for better contraceptive services. Information on the level of unsafe abortion and postabortion complications can be used to help focus public attention on this issue. In addition, it can help the government design improved postabortion care services (including the provision of contraceptive services as part of postabortion care), and programs to educate women and couples about legal provision of abortion and how to obtain the procedure. National data on the incidence of legal abortion is an important indicator of women's access to services under the new criteria and of women's, providers' and the general public's awareness of the abortion law.

\section{DATA AND METHODS}

\section{Data Sources}

The study presented in this article adapted and integrated the design, protocols and data collection approaches of two different methodologies: the Abortion Incidence Complications Methodology (AICM) ${ }^{14,15}$ for estimating abortion incidence and morbidity, and the Prospective Morbidity Methodology ${ }^{16,17}$ for documenting abortion morbidity.

Three data collection efforts were conducted to provide the information needed to estimate abortion incidence in Ethiopia. The first consisted of a survey of a national sample of health facilities that provide postabortion care or safe abortions. The second was a prospective abortionrelated morbidity survey of all postabortion patients treat- ed in the same sample of health facilities during a 28-day period. The third was a survey of a sample of experts knowledgeable about the conditions in which abortion services are provided in Ethiopia. The reference year for this study's estimates is 2008, given that most of the fieldwork was conducted in that year.

Other data sources included the 2000 and 2005 Ethiopian DHS, which provide information on sexual behavior, fertility, contraceptive use, the wantedness of births and unmet need for contraceptive services for a nationally representative sample of women aged 15-49. In addition, we used the data from the 2007 Ethiopian census to estimate the number of women of reproductive age in 2008, nationally and for each region. ${ }^{* 18}$ Furthermore, the annual Health and Health-Related Indicators report provided measures of access to health services essential for this study. ${ }^{19}$

- Health Facilities Survey and prospective morbidity survey. Although these two data collection efforts used the same sample of facilities, they differed in approach and in the information they collected. Details about the number and types of facilities and the sampling frame are described in the preceding article in this issue (page 6). Briefly, of the 898 eligible facilities, 11\% were governmental hospitals, $4 \%$ private or nongovernmental organization (NGO) hospitals, 66\% public health centers, $3 \%$ NGO clinics and $16 \%$ private clinics. For this study, we sampled $100 \%$ of hospitals and NGO clinics, $30 \%$ of public health centers and $40 \%$ of private clinics were sampled. The sample for each of the latter two categories of facilities was drawn within each of 33 strata-three types of facility by the 11 regions of the country. The total number of facilities selected was 393.

Fieldwork was conducted between November 2007 and March 2008. Of the 393 facilities sampled, 344 collected individual patient data for the prospective morbidity survey and 337 collected service and caseload data for the Health Facilities Survey; 347 facilities (88\%) participated in at least one of the two surveys. The reasons for facilities not responding included incorrect contact information, inaccessibility of facilities, closure of the facility and duplicate listing. The survey data were weighted to provide nationally representative estimates, accounting for a facility's probability of selection into the sample and the level of nonresponse, by major region and facility type. ${ }^{\dagger}$ The weighting factor for a given facility type was the inverse of that subgroup's sampling ratio multiplied by the proportion of completed interviews among sampled facilities.

*Detailed age-, sex- and region-specific population data were available from the 2007 Ethiopian census (source: reference 18). We projected the census values forward by one year, applying annual region-specific growth rates from the Ethiopian Central Statistical Agency.

+For 11 of the 33 strata, the numbers of facilities that responded were inadequate to allow for reliable estimation. In these cases, the facilities in that cell were regrouped with a stratum that was similar in facility type and region; analysis weights were appropriately adjusted to account for strata combination. 


\begin{tabular}{|c|c|c|c|c|c|}
\hline \multirow[t]{2}{*}{ Region } & \multicolumn{3}{|c|}{$\begin{array}{l}\text { No. of women treated in health } \\
\text { facilities for abortion complications }\end{array}$} & \multirow[t]{2}{*}{$\begin{array}{l}\text { No. of live } \\
\text { births }\end{array}$} & \multirow[t]{2}{*}{$\begin{array}{l}\text { No. of late } \\
\text { miscarriages§ }\end{array}$} \\
\hline & All* & Miscarriaget & $\begin{array}{l}\text { Induced } \\
\text { abortion } \neq\end{array}$ & & \\
\hline All & 57,964 & 5,357 & 52,607 & $2,964,323$ & 101,083 \\
\hline Tigray & 3,338 & 337 & 3,001 & 163,444 & 5,573 \\
\hline Amhara & 9,242 & 759 & 8,483 & 641,434 & 21,873 \\
\hline Oromiya & 20,310 & 1,724 & 18,586 & $1,185,400$ & 40,422 \\
\hline SNNP & 10,896 & 773 & 10,123 & 618,066 & 21,076 \\
\hline Addis Ababa & 7,233 & 1,077 & 6,156 & 40,564 & 1,383 \\
\hline 4 rural regions ${ }^{* *}$ & 3,274 & 520 & 2,754 & 297,683 & 10,151 \\
\hline 2 urban regionst† & 3,671 & 167 & 3,504 & 17,732 & 605 \\
\hline
\end{tabular}

*Includes both spontaneous and induced abortions. †Calculation assumes that the proportion of women with miscarriages who obtain treatment is the same as the proportion who deliver in facilities. $¥$ The total number treated for any abortion complication minus the number treated for complications of miscarriages. §Miscarriages at 13-21 weeks' gestation, calculated as $3.41 \%$ of all live births. ${ }^{* *}$ Includes Affar, Somali, BenishangulGumuz and Gambela. †tIncludes Harari and Dire Dawa. Note: SNNP=Southern Nations, Nationalities and Peo-

Each respondent of the Health Facilities Survey* was interviewed in person using a structured questionnaire on the facility's postabortion services, including infrastructure and equipment, patient profiles and monthly patient caseloads; separate counts were obtained for the number of inpatients and outpatients. The prospective morbidity survey used a standardized form to obtain data on each woman treated for abortion complications during the 28-day study period; topics included demographic characteristics, reproductive history, vital signs and symptoms, and clinical management. Both surveys obtained data on women who received abortion or postabortion care; in regard to postabortion care, neither survey differentiated between complications resulting from induced abortion and those resulting from miscarriage. The morbidity survey also obtained data on all women who obtained legal abortion procedures in health facilities during the study period.

- Health Professionals Survey. To estimate women's likelihood of experiencing abortion complications and receiving treatment, health professionals who had been selected on the basis of their knowledge and experience were asked about their perceptions regarding abortion-related service provision in Ethiopia. ${ }^{\dagger}$ Priority was given to professionals with experience in rural areas and to obtaining a mix of experts, including nonclinicians. In total, 112 experts were identified, and 80 in-person interviews were completed.

Interviews were conducted using a structured questionnaire that covered a range of topics, including the percentage distribution of women who had had an abortion, by provider type; women's likelihood of experiencing postabortion complications requiring medical care, by provider type; and women's likelihood of obtaining care at

*At each selected facility, a senior staff member who was knowledgeable about the facility's provision of abortion and postabortion care was asked to complete the survey. In many cases, this was the facility manager.

†About seven out of 10 respondents were senior obstetrician-gynecologists, two out of 10 were managers of reproductive health programs, and the rest were policymakers, researchers and others. a health facility, if such a complication occurred. Questions were asked about four subgroups of women-rural poor, rural nonpoor, urban poor and urban nonpoor-to incorporate two key sources of variation in safety of abortion services and access to health care (i.e., poverty and place of residence). Separate questions were asked on the number of outpatients and inpatients for two reference periods: the average month and the past month. Specifying outpatients and inpatients ensures that both are included in the estimate, and obtaining information for two reference periods increases the likelihood of accurate recall and allows for variation from month to month.

\section{Estimating the Incidence of Induced Abortion}

The total annual number of women who had an induced abortion includes those treated at a health facility for complications resulting from induced abortion, those with complications who did not receive care at a health facility (including those who died before they could obtain care) and those without complications. Thus, following the AICM methodology, we first estimated the annual number of women receiving treatment at a health facility for complications of induced abortion, by removing the estimated number of women treated for complications from miscarriages from the number of all women treated for abortion complications. Then, we applied a multiplier to account for the proportion of women who had an induced abortion but did not need treatment, obtained care but not from a health facility or obtained no care at all. Although the prospective data collection provides an estimate of the number of women who had legal abortions in facilities, this count underestimates the total number of women having legal or safe abortions, because it excludes women having legal abortions in places other than the types of facilities included in the sample and those having safe procedures that did not meet legal criteria.

- Women treated for abortion complications. Using data from both the prospective morbidity study and the Health Facilities Survey, we estimated that in 2008, 57,964 women were treated for complications from induced abortions or miscarriages in Ethiopia (Table 1). Annual estimates were calculated for each sampled facility, from all three sources (multiplying the two Health Facilities Survey monthly estimates (past and average month) by 12 , and multiplying the 28-day estimate from the prospective morbidity study by 13.04). Because there was variation among the three estimates, they were averaged to obtain the best estimate of the number of postabortion patients treated annually in each facility.

Because complications of induced abortion and miscarriage are often similar, it is difficult to accurately classify patients by the cause of pregnancy loss, and because stigma and secrecy about induced abortion may lead to underreporting of induced abortion cases, neither the prospective morbidity study nor the Health Facilities Survey obtained information specifically on these two categories of postabortion patients. We therefore used an in- 
direct estimation approach-part of the AICM-to calculate the number of women treated for complications from miscarriages, and then subtracted it from the total number of postabortion patients to obtain the number treated for complications of induced abortion.

We used available data on the biological pattern of miscarriage, established by clinical studies, ${ }^{20,21}$ and assumed that all women having late miscarriages (at 13-21 weeks' gestation) likely require care at a health facility. ${ }^{*}$ Of all recognized pregnancies, an estimated $15 \%$ are miscarriages and $85 \%$ live births; late miscarriages account for $2.9 \%$ of all recognized pregnancies, equal to $3.4 \%$ of all live births (2.9\%/85\%). The number of births in Ethiopia in 2008 was estimated by applying fertility rates from the 2005 DHS to 2008 population estimates of the number of women aged 15-44, nationally and for each region or administrative area. ${ }^{\dagger 18}$ According to these calculations, an estimated 2,964,000 live births and 101,000 late miscarriages occurred in 2008 in Ethiopia (Table 1).

A further adjustment was needed because only a proportion of women requiring treatment for complications of miscarriage have access to a health facility. Nationally, 5.3\% of women deliver at a health facility (from $4 \%$ in some rural regions to $79 \%$ in Addis Ababa). We applied this proxy measure using region-specific values and estimated that 5,350 women are treated each year in health facilities for complications of late miscarriage (Table 1). Therefore, an estimated 52,600 women were treated in 2008 for complications of induced abortion.

- Calculation of the multiplier. We derived the multiplier from information in the Health Professionals Survey. In general, the safer abortion services are in a country, the higher the multiplier, because for every woman receiving postabortion treatment, many have abortions that do not result in complications requiring medical care. Likewise, the less safe abortion services are, the lower the multiplier. Safety is not the only consideration, however. The multiplier also accounts for the accessibility of health facilities and for women's attitudes toward seeking care from facilities. Where facilities are easily accessible, the proportion of women with complications who receive treatment will be higher. In poor regions or in underdeveloped areas, by contrast, some of the most seriously affected women may not get the treatment they need. Where women have negative attitudes toward health facilities, for example, as a result of cultural factors or stigma surrounding induced abortion, their likelihood of seeking care from facilities will also be low.

To calculate the proportions of women experiencing abortion complications and needing medical care among all women who obtained an abortion, we first multiplied the estimated proportions from the Health Professionals Survey of women obtaining induced abortions by the proportion who experienced complications needing treatment in a facility, for each provider type, for each of the four geographic-economic subgroups. We estimated the proportion of women with complications who were likely to obtain care from a health facility for each subgroup, using information from the Health Professionals Survey and external data on access to health care from the Ministry of Health and other studies. ${ }^{\ddagger 19,22}$ For each subgroup, the proportion experiencing serious complications was multiplied by the proportion expected to get care at a facility for these complications. The resulting four proportions were weighted by the relative size of the groups nationally and summed to arrive at a multiplier for the country. We estimated that about $14 \%$ of all women having induced abortions (legal or illegal) were likely to receive treatment for complications. The national multiplier is the inverse of this proportion, 7.3-that is, for approximately every seven women who had an induced abortion, one was treated for complications in a facility.

\section{Calculating Indicators of Abortion and Unintended Pregnancy}

- Abortion measures. Following the approach of the AICM indirect estimation methodology, we calculated a range of estimates to represent variation around the central estimate (which was based on the multiplier). We defined a low and high estimate by subtracting or adding one to the multiplier, respectively; therefore, the three multipliers used were 6.3, 7.3 and 8.3. We calculated the total number of induced abortions in a year as the product of each multiplier and the annual number of women treated in health facilities for complications of induced abortion. Abortion rates and ratios were calculated for all three multipliers. National and regional results are presented; however, because of their small number of postabortion patients, certain regions were grouped together. ${ }^{\S}$

*It is unlikely that women who miscarried in the first trimester would seek medical care from the facilities surveyed (i.e., hospitals and higher-level health centers). It is more likely that they would seek care from lower-level facilities, non-facility-based trained providers or untrained providers. As a result, we did not categorize or exclude any of the postabortion cases treated in facilities as first-trimester spontaneous abortion cases. In addition, based on the WHO classification of spontaneous losses at 22 or more weeks' gestation as fetal deaths rather than as spontaneous abortions, we did not exclude any postabortion cases treated in facilities as spontaneous abortion cases of this gestational age.

†To calculate national and regional estimates of the number of births for 2008 , we multiplied the number of women aged 15-44 in 2008 by the general fertility rate, calculated from the $2005 \mathrm{DHS}$

‡Health Professionals Survey respondents reported extremely high probabilities of serious complications of induced abortion (43-93\%, by subgroup) for women expected to obtain care at a health facility. However, national HMIS data show that much lower proportions of households have access to health care. The following revised estimates of proportions used in this study reflect the average level of health care access measured by the HMIS data, whereas the relative differences among subgroups reflect the data from the Health Facilities Survey-16\% for the rural poor, $30 \%$ for the rural nonpoor, $40 \%$ for the urban poor and $75 \%$ for the urban nonpoor. The proportions expected to receive postabortion care are assumed to be higher than the proportions obtaining primary health care in the general population, due in part to the strong national initiatives to improve access to postabortion care in recent years (source: reference 22). We rely on the Health Professionals Survey for the other two components of the multiplier (distribution by type of abortion provider and the probability of having a complication) because these are much less likely to suffer from this type of upward bias.

$\S$ Four rural regions (Affar, Benishangul-Gumuz, Gambela and Somali) were grouped. Together, these regions account for $9 \%$ of all women aged 15-44; Somali accounts for about three-fifths of this group. Also, two urban regions (Harari and Dire Dawa) were grouped. These two areas account for $1 \%$ of all women aged $15-44$; they were one region until about 17 years ago, when they were split into two separate city administrations. 
TABLE 2. Indicators of availability of postabortion and abortion services, by type of facility

Indicator

All Public Public Private

$(\mathrm{N}=898)$ hospital health or NGO

$(\mathrm{N}=94) \quad$ center facility $\neq$

$(\mathrm{N}=597) \quad(\mathrm{N}=207)$

$\%$ of facilities providing postabortion services

82

98

79

84

$\%$ of facilities providing abortion services

during 4-week fieldwork period

$\geq 1$ procedures

0 procedures

Postabortion care: annual caseload

Average no. of women treated per facility offering

postabortion care*

Total no. of women treated for abortion complications

$\begin{array}{rrrr}43 & 60 & 34 & 61 \\ 7 & 16 & 7 & 2 \\ 79 & 219 & 50 & 84 \\ 57,964 & 20,198 & 23,317 & 14,448 \\ 264 & 80 & 54 & 692 \\ 102,818 & 4,771 & 10,866 & 87,181\end{array}$

Legal abortion service: annual caseload

Average no. of women obtaining legal abortion

procedures per facility offering this care $\dagger$

Total no. of women obtaining legal abortion procedures

*Includes spontaneous and induced abortion complications. †Caseload averages exclude facilities that reported no patients during the 28 -day fieldwork period. Includes hospitals and clinics. Note: All data in this and subsequent tables are weighted. centers and private or NGO facilities ( 50 and 84 per site, respectively). Government hospitals cared for about onethird of all postabortion patients (not shown); because public health centers were much more numerous, they treated a greater proportion of all such patients (40\%).

According to the prospective morbidity data, $50 \%$ of all facilities provided legal abortion services (not shown); of those, $86 \%$ provided one or more procedures in the fourweek study period. Greater proportions of public hospitals and private or NGO facilities than of public health centers provided safe abortion services (76\% and 63\%, respectively, vs. $41 \%$ ); the proportions providing one or more abortion procedures during the period were 60\%, $61 \%$ and $34 \%$, respectively (Table 2). Overall, each facility that provided abortion services during the study period conducted an average of 264 procedures per year; the annual caseload was much lower in the public sector $(54$ per health center and 80 per hospital) than in the private sector (692 per private or NGO facility).

\section{Abortion Morbidity}

- Estimating unintended pregnancy. To calculate numbers and rates of unintended pregnancy, we first calculated the number of unplanned births by applying the proportion of births that are unplanned (mistimed or unwanted at the time of conception) from the 2005 DHS to the estimated total annual number of live births in 2008. A model-based approach derived from clinical studies of pregnancy loss by gestational age was employed to estimate the number of miscarriages. * We applied approximate parameters from this source (that pregnancy losses are equal to approximately $20 \%$ of the number of live births and $10 \%$ of the number of induced abortions) to the estimated numbers of births and abortions, to estimate the total number of miscarriages occurring in the country. Combining the numbers of unintended births, induced abortions and miscarriages of pregnancies that were unintended yielded an estimate of the overall total number of unintended pregnancies for 2008. We then calculated the rate of unintended pregnancies per 1,000 women of reproductive age and the proportion of pregnancies that were unintended.

\section{RESULTS}

\section{Provision of Postabortion and Legal Abortion Services}

Eighty-two percent of sampled facilities reported offering postabortion services (Table 2). Almost all public hospitals (98\%) provided postabortion care; smaller proportions of other facilities (79\% of public health centers and $84 \%$ of private or NGO facilities) did so. Public hospitals-which have the largest capacity of all facility types-treated an average of 219 postabortion patients per facility per year; the average annual caseload was much lower in public health

*We use the term "miscarriage" to refer to all spontaneous fetal losses, including stillbirths. Clinical studies generally document miscarriages starting from the fourth, fifth or sixth week of gestation; miscarriages before the sixth week are not typically included in existing studies and are not part of the pregnancy estimates presented here.
The 2008 national rate of women treated in health facilities for abortion complications was 3.5 per 1,000 women aged 15-44 (not shown); the annual rate of women treated in health facilities for complications from induced abortion was 3.2. The rates in Addis Ababa (6.7) and in the combined two urban regions (25.4) were higher than the national average, likely because of better access to health facilities; urban facilities serving women who reside in their cities' environs, spuriously inflating the rates; higher levels of abortion due to an increased likelihood of unmarried young women becoming sexually active and having an unintended pregnancy as the age at marriage rises; and a high prevalence of prostitution. The rate was lower than average in Amhara and the combined four rural regions (1.8 and 2.2, respectively), and was close to the national average in the remaining three regions (3.0-3.2).

The majority of abortions among nonpoor women were likely performed by trained health professionals, such as physicians, clinical officers, nurses or midwives (an estimated $88 \%$ of urban women and $65 \%$ of rural women; not shown). Among poor women, about $57 \%$ of urban women and $36 \%$ of rural women are considered likely to obtain their abortions from trained professionals-which is understandable given that they may be less likely than more affluent women to know about safe providers and to be able to afford a safe procedure, and because the availability of trained providers in rural areas is much lower than in urban areas. ${ }^{23}$ However, complications may result from procedures carried out by trained providers who have little experience or who work in unhygienic settings. In addition, a substantial proportion of abortions (especially among rural and urban poor women) entail a high risk of complications because they are carried out by informal and untrained providers (traditional healers, lay practitioners, pharmacists or the women themselves).

The results suggest that the unmet need for postabor- 
tion care remains large. Health professionals estimated that $58 \%$ of all women who have abortions experience serious complications, and of those, only $24 \%$ receive treatment at a health facility; the remainder need but do not receive postabortion care from a facility considered to have the capacity to offer such services. It is possible that some women receive care from an individual private health professional (outside of a facility), but there is no information on how many women are in this situation.

\section{Incidence of Induced Abortion}

By applying the medium multiplier to the estimated number of women receiving treatment for complications of induced abortion, we estimated that 382,000 induced abortions occurred in Ethiopia in 2008 (Table 3); the low estimate was 330,000, and the high estimate was 435,000. The medium national estimate of the 2008 abortion rate was 23 per 1,000 women aged 15-44 (Table 4); the estimates ranged from 20 to 26 per 1,000. An estimated 11-15 abortions occurred per 100 live births, depending on the multiplier used; the medium estimate of 13 per 100 live births means that nationally there is about one abortion for every eight births.

Abortion incidence varied widely across regions. The medium estimated abortion rate ranged from 13-16 per 1,000 women in the four rural regions combined and Amhara to 184 per 1,000 women in the two urban regions combined. The abortion rate in Addis Ababa (49 per 1,000 ) was twice the national average-likely the result of many factors, among them a strong motivation on the part of couples to have small families that is not matched by a sufficiently high level of contraceptive use. In 2005, the average wanted family size was 1.2 children, the total fertility rate was 1.4 , and $45 \%$ of married women used modern contraceptive methods. ${ }^{9}$ The age at first marriage increased between 2000 and 2005, with a large proportion of women being unmarried into their mid-20s, increasing the likelihood that they would become sexually active and at risk of unintended pregnancy. ${ }^{9}$ The extremely high abortion rate in the two small urban regions is most likely explained by the fact that they are accessible, commercial centers, which have a large network of private health care providers serving women in surrounding areas.

As with the abortion rate, the abortion ratio varied across regions. The medium estimate was lowest in the combined four rural regions and Amhara (7-10 per 100 live births), and highest in Addis Ababa and the combined two urban regions (110-144 per 100 live births). The factors mentioned above as contributing to the high abortion

*On the basis of the Health Professionals Survey, we estimate that $42 \%$ of all women having abortions-or 161,000—do not experience any serious complications and have safe abortions. From our study, we estimated that 103,000 legal abortions were provided in health facilities. The difference between these two numbers $(58,000)$ is the number of safe abortions being provided outside the categories of health facilities surveyed in the prospective morbidity study. This study does not provide information on whether these abortions are legal or not. In addition, it is possible that a small proportion of the 103,000 legal abortions had complications, and that these were treated in health facilities.
TABLE 3. Number of women aged 15-44 treated in health facilities for complications of induced abortion; and estimated total number of induced abortions, by multiplier accounting for women not treated in a health facility for complications of abortion, according to region

\begin{tabular}{|c|c|c|c|c|}
\hline \multirow[t]{2}{*}{ Region } & \multirow{2}{*}{$\begin{array}{l}\text { No. of women treated } \\
\text { in health facilities } \\
\text { for complications of } \\
\text { induced abortion }\end{array}$} & \multicolumn{3}{|c|}{ Estimated no. of induced abortions } \\
\hline & & 6.3 & 7.3 & 8.3 \\
\hline All & 52,607 & 329,843 & 382,450 & 435,056 \\
\hline Tigray & 3,001 & 18,815 & 21,816 & 24,817 \\
\hline Amhara & 8,483 & 53,188 & 61,670 & 70,153 \\
\hline Oromiya & 18,586 & 116,537 & 135,123 & 153,710 \\
\hline SNNP & 10,123 & 63,469 & 73,592 & 83,715 \\
\hline Addis Ababa & 6,156 & 38,600 & 44,756 & 50,912 \\
\hline 4 rural regions* & 2,754 & 17,265 & 20,019 & 22,772 \\
\hline 2 urban regionst & 3,504 & 21,969 & 25,473 & 28,977 \\
\hline
\end{tabular}

*Includes Affar, Somali, Benishangul-Gumuz and Gambela. HIncludes Harari and Dire Dawa. Note: SNNP=Southern Nations, Nationalities and Peoples.

rates likely account for these high abortion ratios as well. In the case of Addis Ababa, the high abortion ratio is also likely affected by the area's very low level of fertility. ${ }^{9}$

\section{Estimated Number of Legal Abortion Procedures}

According to our estimate based on the medium multiplier (382,000), the approximately 103,000 legal abortions provided by surveyed health facilities in 2008 accounted for $27 \%$ of all induced abortions that year (not shown). Nationally, the incidence of facility-based legal abortion was six per 1,000 women aged 15-44, ranging from almost zero in the combined four rural regions to 41-46 per 1,000 in Addis Ababa and the combined two urban regions. From the Health Facilities Survey and Health Professionals Survey, we infer that an additional 15\% (58,000) of all induced abortions in Ethiopia are safely and possibly legally provided by health professionals outside of the surveyed categories of health facilities.*

\section{Estimated Incidence of Unintended Pregnancies}

Nationally, the proportion of births that were reported to be unplanned (unwanted or mistimed) declined slightly, from 37\% in 2000 to $35 \%$ in 2005 (Table 5). Substantial

\begin{tabular}{|c|c|c|c|c|c|c|}
\hline \multirow[t]{2}{*}{ Region } & \multicolumn{3}{|c|}{ Abortion rate } & \multicolumn{3}{|c|}{ Abortion ratio } \\
\hline & 6.3 & 7.3 & 8.3 & 6.3 & 7.3 & 8.3 \\
\hline All & 19.9 & 23.1 & 26.2 & 11 & 13 & 15 \\
\hline Tigray & 19.5 & 22.6 & 25.7 & 12 & 13 & 15 \\
\hline Amhara & 13.8 & 16.1 & 18.3 & 8 & 10 & 11 \\
\hline Oromiya & 20.1 & 23.3 & 26.5 & 10 & 11 & 13 \\
\hline SNNP & 18.8 & 21.8 & 24.8 & 10 & 12 & 14 \\
\hline Addis Ababa & 42.1 & 48.8 & 55.5 & 95 & 110 & 126 \\
\hline 4 rural regions* & 11.3 & 13.1 & 14.9 & 6 & 7 & 8 \\
\hline 2 urban regionst & 159.0 & 184.4 & 209.8 & 124 & 144 & 163 \\
\hline
\end{tabular}

*Includes Affar, Somali,Benishangul-Gumuz and Gambela. Includes Harari and Dire Dawa.Notes:SNNP=Southern Nations, Nationalities and Peoples. The abortion rate is the number of induced abortions per 1,000 women aged 15-44 per year. The abortion ratio is the number of induced abortions per 100 live births. 


\begin{tabular}{|c|c|c|c|c|c|c|}
\hline \multirow[t]{2}{*}{ Region } & \multicolumn{3}{|l|}{2000} & \multicolumn{3}{|l|}{2005} \\
\hline & $\begin{array}{l}\text { All } \\
\text { unplanned }\end{array}$ & Unwanted & Mistimed & $\begin{array}{l}\text { All } \\
\text { unplanned }\end{array}$ & Unwanted & Mistimed \\
\hline All & 37 & 17 & 20 & 35 & 16 & 19 \\
\hline Tigray & 29 & 11 & 18 & 17 & 6 & 11 \\
\hline Amhara & 42 & 22 & 20 & 31 & 14 & 17 \\
\hline Oromia & 37 & 17 & 20 & 42 & 20 & 23 \\
\hline SNNP & 35 & 14 & 21 & 39 & 19 & 20 \\
\hline Addis Ababa & 47 & 29 & 18 & 44 & 23 & 21 \\
\hline 4 rural regions* & 20 & 11 & 9 & 10 & 4 & 6 \\
\hline 2 urban regionst & 33 & 15 & 18 & 20 & 8 & 12 \\
\hline
\end{tabular}

*Includes Affar, Somali, Benishangul-Gumuz and Gambela. Includes Harari and Dire Dawa. Notes: SNNP= Southern Nations, Nationalities and Peoples. Percentages are based on live births in the five years before interview. Sources: References 7 and 8.

declines occurred in the levels of unplanned births in Tigray and Amhara, as well as in the two combined groups of regions. On the other hand, the levels of unplanned births in Oromiya and the State of Southern Nations, Nationalities and Peoples-which were already high in 2000, increased moderately by 2005 . In Addis Ababa, the proportion of births that were unplanned declined slightly, but remained the highest in the country in 2005 (44\%).

The overall pregnancy rate in Ethiopia in 2008 was estimated to be 242 per 1,000 women (Table 6). By combining induced abortions, unplanned births and unplanned pregnancies that ended in miscarriage, we estimated the rate of unintended pregnancy in 2008 at 101 per 1,000 women. The rate of unintended pregnancy was extremely high in the combined two urban areas (233 per $1,000)$. However, the rate was lower than the national average in Addis Ababa and Amhara (77-80 per 1,000), and much lower than average in Tigray $(58$ per 1,000$)$ and the combined four rural regions (38 per 1,000). Overall, 42\% of all pregnancies in Ethiopia in 2008 were unintended. The proportion of all pregnancies that were unintended

TABLE 6. Number of pregnancies, rate of unintended pregnancy, percentage of pregnancies that were unintended and estimated pregnancy rate, by region, 2008

\begin{tabular}{lllll} 
Region & $\begin{array}{l}\text { No. of } \\
\text { pregnancies* }\end{array}$ & $\begin{array}{l}\text { Rate of } \\
\text { unintended } \\
\text { pregnancyt }\end{array}$ & $\begin{array}{l}\text { \% of } \\
\text { pregnancies } \\
\text { that are } \\
\text { unintended }\end{array}$ & $\begin{array}{l}\text { Pregnancy } \\
\text { ratef }\end{array}$ \\
\hline All & $\mathbf{3 , 9 7 7 , 8 8 3}$ & $\mathbf{1 0 1}$ & $\mathbf{4 2}$ & $\mathbf{2 4 2}$ \\
Tigray & 220,130 & 58 & 26 & 228 \\
Amhara & 837,558 & 80 & 37 & 218 \\
Oromiya & $1,571,115$ & 129 & 48 & 270 \\
SNNP & 822,630 & 109 & 45 & 244 \\
Addis Ababa & 97,908 & 77 & 72 & 107 \\
4 rural regions§ & 379,241 & 38 & 15 & 248 \\
2 urban regions** & 49,299 & 233 & 65 & 357 \\
\hline
\end{tabular}

*Includes births, abortions and miscarriages. + Number of unplanned births, abortions and miscarriages that resulted from unwanted pregnancies per 1,000 women aged 15-44 per year. $\neq$ Number of pregnancies per 1,000 women aged 15-44 per year. SIncludes Affar, Somali, Benishangul-Gumuz and Gambela. ${ }^{* *}$ Includes Harari and Dire Dawa. Note:SNNP=Southern Nations, Nationalities and Peoples. Sources: Population estimates for 2008 are based on the 2007 census numbers for women aged 15-44 by five-year age-groups, projected forward one year. Age-specific fertility rates obtained from the 2005 DHS were applied to the population of women by five-year age-group in 2008, to estimate the number of births in 2008. The proportion of births that were unplanned (unwanted or mistimed, also from the 2005 DHS) was applied to the total number of births (nationally and by region), to obtain the number of unplanned births in 2008. The number of abortions and miscarriages were estimated by the authors. was much higher than average in Addis Ababa (72\%) and was lower than average in Tigray (26\%) and the combined four rural regions (15\%).

\section{DISCUSSION}

This study provides the first country-wide estimate of the incidence of induced abortion in Ethiopia. An estimated 382,000 abortions occurred in 2008, and the annual abortion rate was 23 per 1,000 women aged 15-44. This rate is much lower than WHO's estimated rate for 2003 for Eastern Africa (39 per 1,000), the subregion within which Ethiopia falls, and somewhat lower than the rate for the whole of Africa (29 per 1,000). ${ }^{24}$

Patterns of reproductive behavior in Ethiopia support our findings on abortion incidence. The proportion of all women aged 15-49 accepting a contraceptive method has increased over recent years (from 22\% in 2001-2002 to $34 \%$ in 2006-2007). ${ }^{* 19}$ The overall contraceptive prevalence rate among married women aged 15-49 also increased, from $8 \%$ in 2000 to $15 \%$ in 2005 ; the proportion using modern methods increased from $6 \%$ to $14 \%$ during that time. By 2005, a more widespread desire for smaller families, combined with a low level of contraceptive use, resulted in a very high level of unmet need for contraception: Thirty-four percent of married women aged 15-49 were fecund and did not want a child soon or wanted no more children, but were not using a method. In addition, 14\% of unmarried women aged 15-24 in 2005 had initiated sexual activity; in a society with a strong social stigma against premarital sexual activity, few such women are likely to visit clinics to obtain contraceptives, leaving themselves at risk of out-of-wedlock pregnancy and abortion. ${ }^{25}$

Our estimates of abortion incidence for the different regions in Ethiopia are plausible, given regional levels of fertility and contraceptive use. In the capital city, Addis Ababa, the high abortion rate is consistent with its extremely low TFR (1.4 in 2005), and desired family size (1.2 in 2005), and with its level of current contraceptive use; even though modern method use is moderately high and is higher than the level in all other regions (45\% among married women of reproductive age), it is too low to explain such a low TFR. ${ }^{8}$ Other factors probably also contribute to the high abortion rate in Addis Ababa: For example, it is likely that women from surrounding areas obtain abortion services in the capital city, where such services are more available. The extremely high abortion rate in the two grouped urban regions is consistent with their below average TFRs (3.8 in Harari and 3.6 in Dire Dawa), their relatively low contraceptive prevalence rates (29-32\% among married women of reproductive age) and some special factors that characterize these two cities (i.e.,

* Important differences between the proportion accepting a contraceptive method and the contraceptive prevalence rate include the fact that an unknown proportion of contraceptive supplies that are distributed are not used; and that women who accept a method may discontinue use soon after. 
having extensive private sector health care services and being easily accessible to women in neighboring regions). The much lower abortion rates in other regions are also consistent with their higher TFRs (5.0-6.0) and their lower levels of modern method use (3-16\%). More research is needed to better understand the wide regional variations in abortion incidence.

The large and growing gap between actual fertility and women's average preferred family size (a difference of 0.9 children in 2000 and of 1.4 children in 2005) $)^{8}$ suggests that the abortion rate will likely increase in the next few years if modern contraceptive use does not increase sharply. Consistent with this finding, both the proportion of recent births that are unplanned and the proportion of married women with unmet need are very high and changed little between 2000 and 2005.

Given the lack of nationally representative information on abortion-related services before 2005, no definitive conclusions can be drawn about overall trends in abortion incidence from the data presented here. These data, however, do provide a baseline for assessing trends in future years. In addition, they can provide some indication of the impact of the reform of the abortion law and its implementation on the provision of legal abortion services in health facilities over the three years since 2005. Prior to the reform, abortion was very restricted, and thus it can be assumed that very few legal procedures were performed before 2005. In 2008, an estimated 103,000 women obtained legal abortions in health facilities. It is likely that much of the increase in legal abortion services was facilitated by law reform and the 2006 guidelines, as well as by the substantial efforts on the part of the public, NGO and private sectors to implement the provision of safe abortion services, following the regulatory changes. ${ }^{26}$ Because $80 \%$ of public health centers-which are more accessible to rural women-provide postabortion care, but only 34\% provide legal abortion services, a reorientation of these facilities to increase their provision of safe, legal abortion services is an important step toward addressing the disproportionately poor access of rural women.

In addition, these results do not allow us to draw definitive conclusions about overall trends in the safety of abortion, given the lack of information on the number of safe clandestine abortions that occurred prior to 2005 or the number that may still be occurring. Still, as training in abortion provision continues to expand throughout the country among both physicians and midwives, as it has in the past four years, it is likely that the safety of clandestine abortions will continue to improve. Some supporting evidence is provided by a study in one major university hospital in Addis Ababa that found a decline in the ratio of abortion complications cases to live births after the law changed in 2005, suggesting that abortion morbidity declined as a result of the law reform. ${ }^{27}$

Unsafe abortion continues to be an important issue: Health professionals estimated that more than half of all abortions in 2008 resulted in complications that needed care. In addition, the unmet need for postabortion care remains large: Only about a quarter of women who need treatment for induced abortion complications receive care at health facilities that provide postabortion services. Government facilities bear most of the burden of providing postabortion care, serving $75 \%$ of these patients. It is possible that some women with postabortion complications may receive care from providers outside of these facilities, but there is no information on the extent to which this may be happening. The scarcity of trained health care professionals at all levels and of health facilities that can provide postabortion care help to explain the low proportion of women receiving postabortion care: There are 25,000 people for every physician or health officer in Ethiopia, and about 4,000 people for every nurse. ${ }^{19}$ The low levels of skilled attendance at birth and of health care utilization in general support the estimated low proportion receiving postabortion care.

\section{Policy and Program Implications}

Our findings have several implications for policies and programs. The importance of meeting the need for contraceptive services cannot be overemphasized, given the high level of unmet need for contraception among married women and the very high unintended pregnancy rate. In-depth local studies can provide useful guidance on how best to inform and reach women who need contraceptive services, but they also highlight gaps in postabortion care (e.g., only one in four women who have unsafe abortion complications are estimated to receive this care in this study area). ${ }^{28}$ However, it is important to emphasize that the broader constraints within the health care system are linked to these gaps in provision of reproductive services. Improvement in the overall capacity to provide health care services is essential to meet the need for reproductive health services.

Significant progress has been achieved in implementing access under the expanded indications of the 2005 law reform, given the high proportions of health facilities that are providing legal and safe abortion services; however, the proportion of public health centers doing so is much lower than the proportion of public hospitals or private and NGO facilities. There is great need to expand provision of affordable public sector services. In particular, access must be improved for rural women, who represent $82 \%$ of all women of reproductive age ${ }^{8}$ and who are more likely than urban women to experience unsafe abortion and its consequences. ${ }^{29}$ However, it is important to acknowledge substantial expansions in the public health sector in recent years; For example, the number of health extension workers almost doubled from 8,900 in 2005 to 17,600 in $2006 .{ }^{19}$ Further research is needed to measure change in services and related factors to better inform planning and implementation.

A number of medication abortion products are being considered for official registration and government approval. Approval of one or more of these products could improve access to safe abortion care overall, especially for rural woman. Expanding training in comprehensive abortion care is another important step toward improving ac- 
cess to safe abortion services. These various strategies will need to be implemented simultaneously to reduce unintended pregnancy and unsafe abortion and their negative impact on women's health and survival.

\section{REFERENCES}

1. Ethiopian Ministry of Health, Technical and Procedural Guidelines for Safe Abortion Services in Ethiopia, Addis Ababa, Ethiopia: Ministry of Health, 2006.

2. World Health Organization (WHO), Maternal Mortality in 2005: Estimates Developed by WHO, UNICEF, UNFPA and the World Bank, Geneva: WHO, 2005.

3. WHO, Unsafe Abortion: Global and Regional Estimates of the Incidence of Unsafe Abortion and Associated Mortality in 2003, fifth ed., Geneva: WHO, 2007.

4. Berhan $Y$ and Abdela A, Emergency obstetric performance with emphasis on operative delivery outcomes: does it reflect the quality of care? Ethiopian Journal of Health Development, 2004, 18(2):96-106.

5. Mekbib T, Gebrehiwot Y and Fantahun M, Survey of unsafe abortion in selected health facilities in Ethiopia, Ethiopian Journal of Reproductive Health, 2007, 1(1):28-43.

6. Ethiopian Society of Obstetricians and Gynecologists (ESOG), A Data Base on Abortion Literature Review, Addis Ababa, Ethiopia: ESOG, 2000.

7. Central Statistical Agency (Ethiopia) and ORC Macro, Ethiopia Demographic and Health Survey, 2000, Addis Ababa, Ethiopia: Central Statistical Agency; and Calverton, MD, USA: ORC Macro, 2001

8. Central Statistical Agency (Ethiopia) and ORC Macro, Ethiopia Demographic and Health Survey, 2005, Addis Ababa, Ethiopia: Central Statistical Agency; and Calverton, MD, USA: ORC Macro, 2006.

9. Sibanda A et al., The proximate determinants of the decline to below replacement fertility in Addis Ababa, Ethiopia, Studies in Family Planning, 2003, 34(1):1-7.

10. Gurmu E and Mace R, Fertility decline driven by poverty: the case of Addis Ababa, Ethiopia, Journal of Biosocial Science, 2008, 40(3): 339-358.

11. Seifu A, Fantahun M and Worku A, Reproductive health needs of out-of-school adolescents: a cross-sectional comparative study of rural and urban areas in northwest Ethiopia, Ethiopian Journal of Health Development, 2006, 20(1):10-17.

12. Taffa N et al., Psychosocial determinants of sexual activity and condom use intention among youth in Addis Ababa, Ethiopia, International Journal of STD \& AIDS, 2002, 13(10):714-719.

13. Adamu R, Samuel M and Ingidashet S, Patterns and correlates of sexual initiation, sexual risk behaviors, and condom use among secondary schools in Ethiopia, Ethiopian Medical Journal, 4l(2):163-177.

14. Singh S and Wulf D, Estimated levels of abortion in six Latin American countries, International Family Planning Perspectives, 1994, 20(1): 4-13.

15. Singh S et al., The incidence of induced abortion in Uganda, International Family Planning Perspectives, 2005, 31(4):183-191.

16. Jewkes R et al., Prevalence of morbidity associated with abortion before and after legalization in South Africa, BMJ, 2002, 324(7348): 1252-1253.

17. Gebreselassie $\mathrm{H}$ et al., The magnitude of abortion complications in Kenya, British Journal of Obstetrics and Gynaecology, 2005, 112(9): 1229-1235.

18. Federal Democratic Republic of Ethiopia Population Census Commission, Summary and Statistical Report of the 2007 Population and Housing Census-Population Size by Age and Sex, Addis Ababa, Ethiopia: Population Census Commission, 2008

19. Ethiopian Ministry of Health, Planning and Programming Department, Health and Health-Related Indicators, Addis Ababa, Ethiopia: Ministry of Health, 2007.
20. Harlap S, Shiono PH and Ramcharan S, A life table of spontaneous abortions and the effects of age, parity and other variables, in: Hook EB and Porter I, eds., Human Embryonic and Fetal Death, New York: Academic Press, 1980, pp.145-158.

21. Bongaarts J and Potter RG, Fertility, Biology and Behavior: An Analysis of the Proximate Determinants, New York: Academic Press, 1983.

22. Fetters T, Tesfaye $S$ and Clark K, An assessment of postabortion care in three regions in Ethiopia, 2000 to 2004, International Journal of Gynecology and Obstetrics, 2008, 101(1):100-106.

23. Unpublished data from the 2008 Guttmacher Health Professionals Survey of Ethiopia.

24. Sedgh G et al., Induced abortion: estimated rates and trends worldwide, Lancet, 2007, 370(9595):1338-1345.

25. Special tabulations of data from the 2000 and 2005 DHS surveys.

26. Gebreselassie H and Fetters T, Responding to Unsafe Abortion in Ethiopia: A Facility-Based Assessment of Postabortion Care Services in Public Health Sector Facilities in Ethiopia, Chapel Hill, NC, USA: Ipas, 2002

27. Gebrehiwot $Y$ and Liabsuetarjul T, Trends of abortion complications in a transition of abortion law revisions in Ethiopia, Journal of Public Health, 2008, 31(1):81-87.

28. Hemmings J et al., Ethiopian Women's Perspectives on Reproductive Health: Results from a PEER Study in the Guraghe Zone, London: Options Consultancy Services, 2008

29. Special tabulations of the 2008 Health Professionals Survey of Ethiopia.

\section{RESUMEN}

Contexto: El aborto inseguro es un importante problema de salud en Etiopía; sin embargo, no existe un estudio cuantitativo sobre la incidencia del aborto a nivel nacional. En 2005, se revisó el código penal para ampliar las indicaciones bajo las cuales el aborto inducido es legal. Es importante medir la incidencia del aborto inducido legal e ilegal, después del cambio en la ley.

Métodos: En 2007-2008, se realizó en Etiopía una encuesta representativa a nivel nacional, de una muestra de 347 instituciones de salud que proporcionan servicios de postaborto o aborto seguro, y se aplicó una encuesta a 80 profesionales conocedores de la prestación de servicios de aborto. Se usaron técnicas de estimación indirecta para calcular la incidencia del aborto inducido. Las tasas de aborto, las razones de aborto y las tasas de embarazos no planeados se calcularon para el país y para las principales regiones.

Resultados: Se estima que, en 2008, ocurrieron 382,000 abortos inducidos en Etiopía; y se dio tratamiento a 52,600 mujeres por complicaciones derivadas de dichos abortos. Las instituciones de salud proporcionaron unos 103,000 procedimientos legales-sólo un $27 \%$ de todos los abortos en el país. A nivel nacional, la tasa anual de abortos fue de 23 abortos por 1,000 mujeres en edades de 15-44, y la razón de aborto fue de 13 abortos por 100 nacidos vivos. La tasa de aborto en Adís Abeba (49 por 1,000 mujeres) fue el doble que la tasa nacional. En general, alrededor del 42\% de los embarazos fueron no planeados; y la tasa de embarazos no planeados fue de 101 por 1,000 mujeres.

Conclusiones: El aborto inseguro es todavía común y representa un alto costo para las mujeres en Etiopía. Para reducir las tasas de embarazos no planeados y de aborto inseguro, se necesita un mayor acceso a servicios de anticoncepción y de aborto seguro de alta calidad. 


\section{RÉSUMÉ}

Contexte: L'avortement non médicalisé représente un sérieux problème de santé en Éthiopie. Il n'existe cependant aucune étude nationale quantitative de l'incidence de l'avortement. En 2005, le code pénal a été révisé pour élargir les indications d'admission légale de l'IVG. Il importe de mesurer l'incidence de l’IVG légale et illégale après la réforme de la loi.

Méthodes: Une étude nationalement représentative d'un échantillon de 347 établissements de santé offrant des services de soins post-avortement et d'avortement médicalisé et une enquête auprès de 80 professionnels informés sur la question de l'avortement dans le pays ont été menées en Éthiopie en 2007-2008. Les techniques d'estimation indirecte ont été appliquées pour calculer l'incidence de l'IVG. Les taux et quotients d'avortement et les taux de grossesse non planifiée ont été calculés pour le pays et ses régions principales.

Résultats: On estime à 382.000 le nombre d'IVG pratiquées en Éthiopie en 2008; 52.600 femmes ont été traitées pour cause de complications. Les IVG légales pratiquées dans les établissements de santé du pays sont estimées à 103.000, soit $27 \%$ de la totalité. Le taux d'avortement annuel national est de 23 pour mille parmi les femmes âgées de 15 à 44 ans, pour un

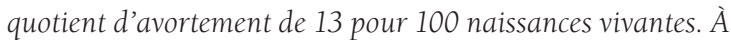
Addis-Abeba, le taux d'avortement (49 pour mille) est deux fois le niveau national. Dans l'ensemble, environ $42 \%$ des gros- sesses n'étaient pas planifiées, soit un taux de grossesse non planifiée de 101 pour mille femmes.

Conclusions: L'avortement non médicalisé reste courant et impose un lourd fardeau aux Éthiopiennes. Pour réduire les taux de grossesse non planifiée et d'avortement non médicalisé, un accès accru doit être assuré à des soins contraceptifs et services d'avortement de haute qualité.

\section{Acknowledgments}

The authors thank Solomon Tesfaye, Takele Geressu and Yilma Melkamu for their invaluable contribution to data collection and data processing; the 16 regional coordinators, for their supervision of data collection; Kathryn Anderson Clark and Elena Prada, for research support; Akinrinola Bankole, Gustavo Suarez, Leila Darabi, Saba Kidanemariam and Janie Benson, for reviewing drafts of this article; and Heran Belete, for administrative support throughout the project. The authors also thank the Ministry of Health and the Regional Health Bureaus for endorsing the project and facilitating data collection. They acknowledge the contributions and support of the Ethiopian Public Health Association and the Ethiopian Society of Obstetricians and Gynecologists as collaborating partners on this project. This research was conducted as part of the Consortium for Research on Unsafe Abortion in Africa, supported by the Department for International Development of the United Kingdom. The findings and conclusions expressed are entirely those of the authors.

Author contact: ssingh@guttmacher.org 